\title{
Efectividad del mindfulness en personas con TDAH: estudio de revisión
}

\author{
Virginia Mariño Fontenla Universitat Oberta de Catalunya \\ Pilar Sanz Cervera. Universidad de Valencia \\ $\mathrm{M}^{\mathrm{a}}$ Inmaculada Fernández Andrés. Universidad de Valencia
}

Recepción: 4 de noviembre de 2017 | Aceptado: 10 de noviembre de 2017

Correspondencia: Ma Inmaculada Fernández | m.inmaculada.fernandez@uv.es

iD orcid.org/0000-0003-2808-0949

Citar: Mariño Fontenla, V. Sanz Cervera, P. y Fernández Andrés, MI. (2017). Efectividad del minfulness en personas con TDAH: estudio de revisión. ReiDoCrea, 6, 260-273.

\begin{abstract}
Resumen: El Trastorno por Déficit de Atención con o sin Hiperactividad (TDAH) es un trastorno de origen neurobiológico que se caracteriza por un déficit de atención y dificultades de concentración, que puede cursar con hiperactividad e impulsividad. Este trastorno suele causar efectos negativos en el ámbito escolar, familiar y social. Con el objetivo de paliar estos efectos adversos, el Mindfulness es una intervención basada en la meditación oriental, que se introdujo en la medicina occidental en los años 70, de la mano de Jon Kabat-Zinn, quien desarrolló un programa de reducción del estrés (MBSR). Desde entonces el Mindfulness se ha utilizado en diferentes contextos, incluido el clínico, como tratamiento de diferentes patologías psiquiátricas, demostrando su efectividad en la reducción y mejora de la sintomatología de varias de ellas. En el caso del TDAH, diversos estudios han hecho uso del Mindfulness, obteniendo resultados positivos en cuanto al nivel de atención, regulación emocional, autocontrol, ansiedad y agresividad. En este artículo, en concreto, se revisan 13 estudios que aplican el Mindfulness en niños, adolescentes y adultos diagnosticados con TDAH. A pesar de algunas limitaciones metodológicas, estos estudios corroboran la efectividad del Mindfulness como una alternativa o un complemento al tratamiento de este trastorno.
\end{abstract}

Palabras clave: Trastorno por Déficit de Atención con o sin Hiperactividad (TDAH) | Mindfulness

\section{Effectiveness of mindfulness intervention in people with ADHD: a review study}

Abstract: Attention-deficit/hyperactivity disorder (ADHD) is a neurobiological disorder that is characterized by attention deficit and concentration difficulties which can occur with hyperactivity and impulsivity. This disorder usually causes negative effects in the school, family and social environments. With an aim to alleviating these adverse effects, Mindfulness is an intervention based on oriental meditation, introduced in western medicine in the 1970s by Jon Kabat-Zinn, who developed a stress reduction program (MBSR). Since then, Mindfulness has been used in different contexts, including the clinical, as a treatment of different psychiatric pathologies, demonstrating its effectiveness in reducing and improving the symptoms of several of them. In the case of ADHD, several studies have applied Mindfulness interventions, obtaining positive results in terms of attention level, emotional regulation, self-control, anxiety and aggressiveness. In this article, in particular, we review 13 studies that apply Mindfulness in children, adolescents and adults diagnosed with ADHD. Despite some methodological limitations, these studies corroborate the effectiveness of Mindfulness as an alternative or a complement to the treatment of this disorder.

Keywords: Attention-deficit/hyperactivity disorder (ADHD) | Mindfulness

\section{Introducción}

El Trastorno por Déficit de Atención con o sin Hiperactividad (TDAH) es un trastorno de origen neurobiológico que suele conllevar dificultades en el desarrollo personal, social y académico de las personas que lo sufren. El patrón característico de síntomas, como son el déficit de atención, las dificultades de concentración, distraibilidad, hiperactividad e impulsividad, es persistente e inapropiado para la edad o el nivel de desarrollo del niño y no se explica por otros trastornos del neurodesarrollo u otros trastornos mentales (APA, 2013). 
Hoy en día, el TDAH es uno de los trastornos neuropsiquiátricos más comunes y con mayor aumento en el mundo. Aunque hay discrepancias sobre su prevalencia, a nivel mundial se sitúa entre el 8 y el 12\% (Polanczyk, De Lima, Horta, Biederman y Rohde, 2007). En España afecta entre un 3\% y un $7 \%$ de los niños en edad escolar pero hay pocos datos sobre la prevalencia en edad adulta. Un estudio realizado en 2010, teniendo como criterio diagnóstico las historias clínicas en atención primaria, ofrece una prevalencia del $0.04 \%$, dato excesivamente bajo que hace sospechar que hay un infradiagnóstico de TDAH en adultos.

Este trastorno presenta una alta comorbilidad con otros trastornos. Algunos estudios indican que la mitad de los niños con TDAH suelen tener asociados al menos otros dos trastornos, como pueden ser: el Trastorno Negativista Desafiante (TND), el Síndrome de Tourette, Trastornos de ansiedad, Tics o Dificultades de Aprendizaje como la Dislexia (Jensen, 2001; Pliszka, 1989). Además de la elevada comorbilidad, los niños con TDAH tienen 4 veces más riesgo de sufrir accidentes o lesiones debido a la impulsividad que caracteriza al trastorno.

En el caso de los adultos con TDAH se presenta una alta comorbilidad con otros desórdenes afectivos, como el abuso de estupefacientes, trastornos de ansiedad o desórdenes de la personalidad (Biederman et al., 1997), que pueden conllevar un alto impacto en sus vidas con consecuencias importantes. De hecho, se ha relacionado con un alto porcentaje de divorcios, implicaciones en delitos, desempleo o bajo nivel educativo.

Según los criterios diagnósticos de la última versión del Manual Diagnóstico y Estadístico de los Trastornos Mentales (DSM-5; APA, 2013), en función del síntoma más predominante encontramos tres clasificaciones: presentación predominante con falta de atención, presentación predominante hiperactivo/impulsiva o presentación combinada. Algunos estudios señalan que aquellos niños que poseen una presentación predominante de inatención presentan más problemas en el aprendizaje (Daley y Birchwood, 2010), mientras que los que poseen una presentación predominante de hiperactividad e impulsividad suelen presentar mayores problemas de conducta y de relación social (Greenfield y Fischel, 2005).

Una de las principales novedades del DSM-5 es considerar que este trastorno tiene una continuidad desde la infancia hasta la edad adulta, además de que se considera que la edad de aparición de los síntomas por primera vez pasa de los 7 (DSM-IV; APA, 2000) a los 12 años (APA, 2013). Los datos nos indican que entre el $50 \%$ y el $70 \%$ de los niños diagnosticados con TDAH mantendrán sus síntomas durante la adolescencia y entre el $40 \%$ al $50 \%$ proseguirán con la sintomatología en la edad adulta (Ortiz-Léon y Jaimes-Medrano, 2007).

Respecto a los tratamientos, los más usados y con mayor efectividad demostrada actualmente son: la terapia cognitivo- conductual y la medicación. Esta última se ha convertido en el tratamiento de elección en la mayoría de los casos. No obstante, ambos tratamientos presentan ciertas limitaciones (Van der Oord, Bogels y Peijnenburg, 2012). En el caso de la terapia cognitivo-conductual, los efectos positivos presentan una baja generalización y son limitados a largo plazo. La medicación también tiene efectos positivos solo a corto plazo, además de que entre un $10 \mathrm{y}$ un $20 \%$ de los niños con TDAH no responden positivamente (Greenhill, Halperin y Abikoff, 1999), la adherencia al tratamiento en la adolescencia es baja y puede presentar serios efectos secundarios (Faraone, 2003).

El aumento de niños diagnosticados y el incremento en la venta de medicamentos es un tema controvertido. Respecto al diagnóstico se cuestiona el hecho de que no 
existen pruebas objetivas neuropsicológicas o genéticas, por lo que el diagnóstico parte de una subjetividad en función de los criterios utilizados, las pautas de observación o las pruebas empleadas. Algunas voces críticas consideran que esta subjetividad lleva a diagnosticar de TDAH a niños inquietos, distraídos o cuya conducta responde a una llamada de atención. En cuanto al exponencial incremento de la venta de medicamentos para tratar el TDAH, algunos autores sospechan que, detrás de este aumento en los diagnósticos, subyacen agresivas estrategias comerciales de la industria farmacéutica (Moffitt y Melchior, 2007).

Teniendo en cuenta el aumento progresivo de diagnósticos, la cronicidad y las graves interferencias que puede causar en la vida social y familiar de las personas con TDAH, su atención se ha convertido en un problema de salud pública que requiere la búsqueda de nuevos tratamientos.

Desde los años 80 se vienen desarrollando lo que se ha denominado como terapias de tercera generación. Estas nuevas terapias tienen en cuenta el contexto y las funciones de los fenómenos psicológicos más que su forma, usando estrategias de cambio basadas en la experiencia y en el contexto (Hayes, Luoma, Bond, Masuda y Lillis, 2006). Entre estas terapias de tercera generación encontramos el Mindfulness como una nueva aproximación para la reducción del estrés y para el tratamiento de algunos problemas psiquiátricos (Baer, 2003).

El Mindfulness es una práctica de tradición oriental, mediante la cual dirigimos nuestra atención al momento presente, observando sin juzgar las experiencias de cada momento (Kabat-Zinn, Massion, Kristeller y Peterson, 1992). Bishop et al. (2004) definen esta práctica como: "una autorregulación de la atención, mantenida en la experiencia inmediata, que permite así un mayor reconocimiento de los acontecimientos mentales en el momento presente y que adopta una orientación particular hacia la propia experiencia, caracterizada por la curiosidad, la apertura y la aceptación".

En sus inicios, John Kabat-Zinn, profesor emérito en la Facultad de Medicina de la Universidad de Massachussets, empezó aplicando su Programa de Reducción del Estrés Basado en Mindfulness (MBSR; Kabat-Zinn et al., 1992) para tratar ataques de pánico, dolor crónico y ansiedad. Desde ese momento, el Mindfulness se ha utilizado en diferentes contextos, incluido el clínico, como tratamiento de diferentes patologías psiquiátricas, demostrando su efectividad en la reducción y mejora de la sintomatología en varias de ellas.

Las terapias más utilizadas en este ámbito han sido: 1) la Terapia Cognitiva Basada en Mindfulness (MBTC; Segal, Williams y Teasdale, 2006), eficaz en la reducción de recaídas en casos de depresión; 2) la Terapia de Aceptación y Compromiso (ACT; Hayes et al., 2006) para promover mayor flexibilidad psicológica, mejorando la capacidad de actuar con eficacia según los valores u objetivos personales incluso en situaciones difíciles; y, 3) la Terapia Dialéctico-Comportamental (DBT; Linehan, 2003), efectiva para el tratamiento del trastorno límite de personalidad.

En el año 2003, Baer realizó una revisión empírica sobre intervenciones basadas en mindfulness encontrando efectos moderados en el tratamiento del dolor crónico, comida compulsiva, ansiedad y depresión en adultos, por lo que propuso que el entrenamiento en Mindfulness se clasificara como intervención clínica. Posteriormente, la revisión empírica de estudios llevada a cabo por Keng, Smoski y Robins (2011) sobre el impacto del mindfulness en la salud mental, amplía las conclusiones de Baer (2003). En esta revisión encontraron una correlación positiva entre la práctica, el 
bienestar psicológico de las personas y una mayor regulación emocional y control de la negatividad asociada a diferentes psicopatologías.

El mindfulness es una práctica de autorregulación de la atención y de la emoción (Teasdale et al., 2000) y el TDAH es precisamente un trastorno que conlleva problemas de autorregulación de pensamientos, de la atención y de las emociones, que además puede ir asociado con estrés, ansiedad, depresión y déficits cognitivos. Por ello, considerando los resultados obtenidos en otros estudios, este puede ser un tratamiento alternativo o complementario efectivo para el tratamiento del TDAH.

Diversos trabajos han estudiado los efectos del entrenamiento basado en mindfulness en niños y adolescentes con TDAH y los resultados son prometedores (Bögels, Hoogstad, van Dun, de Schutter y Restifo, 2008; Singh et al., 2010; Van der Oord et al., 2012). Similares resultados se han obtenido en adultos (Hesslinger et al., 2002; Zylowska et al., 2008) y en intervenciones con familias de adolescentes (Singh et al., 2010).

Cabe considerar que los padres de niños y adolescentes diagnosticados con TDAH experimentan mayor estrés y se sienten con una menor capacidad para enfrentarse a su labor como padres. Este alto nivel de estrés eleva la conflictividad en la familia (Johnston y Mash, 2001), por lo que la práctica del mindfulness es una adecuada intervención para paliar estos efectos.

\section{Objetivos o hipótesis}

Teniendo en cuenta las potencialidades de esta prometedora técnica de intervención, el objetivo de este estudio consiste en realizar una revisión teórica de 13 trabajos publicados recientemente, con el objetivo de analizar la efectividad del entrenamiento en mindfulness con personas diagnosticadas de TDAH.

\section{Métodos}

Para realizar la búsqueda bibliográfica se emplearon las bases de datos: PsycINFO, ERIC y PubMed, empleando las palabras clave: "mindfulness", "intervention", "therapy", "treatment", "attention deficit and hyperactivity disorder (ADHD)", tanto en inglés como en español. Para acotar la búsqueda y obtener un número razonable de resultados, restringimos la búsqueda mediante filtros y operadores booleanos (Y/AND, O/OR y NO/NOT) ver tabla 1.

Los artículos incluidos en esta revisión se han seleccionado teniendo en cuenta los siguientes criterios de inclusión:

- Búsqueda limitada al período comprendido entre 2006 y 2016.

- Artículos empíricos publicados en español o en inglés.

- Estudios que aplican intervenciones basadas en mindfulness a personas diagnosticadas con TDAH (niños, adolescentes y adultos).

- Estudios que analizan la efectividad del mindfulness en personas con TDAH y otros trastornos con una comorbilidad elevada con el TDAH, como ansiedad, depresión o trastorno negativista desafiante.

De un total de 37 artículos localizados, se eliminaron aquellos que:

- La intervención no tenía como elemento central el mindfulness.

- Los participantes no tenían diagnóstico de TDAH o el diagnóstico no se especificaba.

- Investigaciones anteriores al 2006 
Tabla 1. Diagrama de búsqueda de artículos utilizados en la investigación

\begin{abstract}
Palabras buscadas

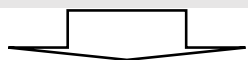

$\mathrm{ADHD}+$

Mindfulness+

Intervention, therapy treatment
\end{abstract}

Base de datos

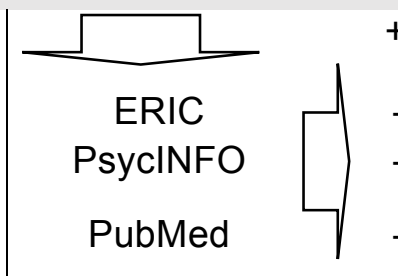

Selección

+ 37, Artículos encontrados

- 10, No grupo TDAH

- 4, No Mindfulness

10, Investigaciones

anteriores 2006

TOTAL

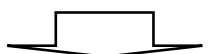

13 artículos utilizados

Para realizar el diagnóstico de TDAH, en todos los estudios se utilizan los criterios del DSM-IV de la Asociación Americana de Psiquiatría (APA, 2000), que define el TDAH como un trastorno del desarrollo neurológico, el cual consta de un patrón persistente de inatención y/o hiperactividad-impulsividad, que obstaculiza el funcionamiento o el desarrollo adecuado del individuo. Se detallan nueve síntomas de inatención y nueve síntomas de hiperactividad-impulsividad, manifiestos antes de los 7 años y presentes en dos o más contextos, que influyen directamente en las actividades sociales y académicas/laborales. Algunos de los estudios utilizan escalas específicas de diagnóstico como la Escala CONNERS para jóvenes (CAARS) o la ECl (Escala del Comportamiento Infantil) -CDI en inglés-, pero en ambos casos se utilizan como un instrumento de medida pretest/postest, y no tanto como instrumento diagnóstico.

Con el objetivo de realizar una comparativa más ajustada, seleccionamos aquellos estudios en los que las intervenciones son adaptaciones modificadas del programa de reducción del estrés (MSBR; Kabat-Zinn et al., 1992) o de la terapia cognitiva basada en mindfulness (MBTC; Segal et al., 2006) y en las que la duración del programa supera al menos las 8 semanas. Hemos descartado estudios en los que la intervención utiliza otros tipos de intervención como el yoga y la meditación trascendental. Tras realizar la búsqueda bibliográfica descrita, se seleccionaron 13 artículos que cumplían con los criterios de inclusión señalados

\section{Resultados}

En la Tabla 2 aparece reflejada toda la información de los 13 artículos seleccionados. Esta tabla incluye: a) los autores del estudio y el año de publicación; b) datos sobre la muestra (tamaño, rango de edad y diagnóstico); c) los instrumentos de evaluación utilizados; d) el tipo de intervención y duración de la misma; e) el diseño de la intervención; y f) los resultados obtenidos.

De todos los estudios que se incluyen en la revisión, 5 se han realizado con personas adultas (Bueno et al., 2015; Janssen et al., 2015; Mitchell et al., 2013; Schoenberg et al., 2014; Smalley et al., 2009), 1 incluye adultos y adolescentes (Zylowska et al., 2008), 1 se realiza exclusivamente con una muestra muy reducida de niños (Carboni, Roach y Fredrick, 2013), y los otros 6 estudios se realizan con niños o adolescentes con un entrenamiento simultáneo con los padres (Haydicky, Shecter, Wiener y Ducharme, 2015; Lo, Wong, Wong, Wong y Yeung, 2016; Meppelink, de Bruin y Bögels, 2016; Singh et al., 2010; Van de Weijer-Bergsma, Formsma, de Bruin y Bögels, 2012; Van der Oord et al., 2012). Los padres de niños con TDAH suelen experimentar altos niveles de estrés y mayor conflictividad en casa, por lo que incluirlos en la intervención suele tener efectos beneficiosos para toda la familia. 
Las intervenciones mindfulness utilizadas en los diferentes estudios difieren según el programa aplicado, aunque prácticamente todos los estudios utilizan versiones modificadas del programa de reducción de estrés (MSBR) del profesor Kabat-Zinn et al. (1992) o de la terapia cognitiva basada en mindfulness (MBTC) de Segal et al. (2006). Los estudios realizados con adolescentes adaptan los programas en función de las necesidades, como el programa Mymind, desarrollado por Bögels et al. (2008) y basado en el MBTC, o el programa MAPs desarrollado por Zylowska, Smalley y Schwartz (2008), el cual está basado en los programas MBRS, MBTC y en la tradición de la meditación vipassana.

Los diferentes estudios también difieren en la duración de las intervenciones, aunque todos alcanzan el mínimo de 8 semanas, extendiéndose en algunos casos hasta las 12 e incluso 17 semanas (Carboni et al., 2013). La duración de las sesiones semanales va desde los 45 minutos a las 3 horas. Algunos programas incluyen CD, bibliografía, informes o formularios para registrar las prácticas y algunos de ellos, aunque no todos, incluyen retiro de un día.

En cuanto a los resultados obtenidos, en 6 de los estudios seleccionados se han encontrado mejoras significativas respecto a la sintomatología TDAH en adultos (Bueno et al., 2015; Janssen et al., 2015; Mitchell et al., 2013; Schoenberg et al., 2015; Smalley et al., 2009; Zylowska et. al., 2008). Ocho de los estudios refieren un decremento en las dificultades atencionales (Bueno et al., 2015; Janssen et al., 2015; Lo et al., 2016; Mitchell et al., 2013, Schoenberg et al., 2014; Van de Weijer-Bergsma, et al., 2012; Van der Oord et al., 2012; Zylowska et al., 2008); y en tres de los estudios se obtienen mejoras respecto al nivel de hiperactividad (Carboni et al., 2013; Lo et al., 2016; Mitchell et al., 2013).

Además de las mejoras en la sintomatología TDAH, algunos estudios tienen en cuenta otras variables importantes. En la investigación de Carboni et al. (2013), aunque no se encuentran mejoras en los síntomas de atención sí se detectó una mejora en la ejecución de las tareas en clase. Zylowska et al. (2008) obtuvieron mejoras en la sintomatología depresiva y de ansiedad, y los sujetos del estudio de Bueno et al. (2015) también manifestaron mejoras en su calidad de vida y mayor control emocional. Otros estudios encontraron diferentes beneficios como mayor autocompasión, una actitud mental más positiva, y una mejora funcional general (Janssen et al., 2015), o mejoras respecto al cumplimiento y obediencia de los menores con respecto a los requerimientos paternos, mejorando las relaciones e interacciones familiares (Singh et al., 2010).

Algunos estudios, además, demuestran la importancia de incluir a las familias en una intervención paralela, mostrando efectos positivos en todos ellos (Haydicky et al., 2015; Lo et al., 2016; Singh et al., 2010; Van de Weijer-Bergsma et al., 2012; Van der Oord et al., 2012). 
Tabla 2. Información relevante de las investigaciones seleccionadas

\begin{tabular}{|c|c|c|c|c|c|}
\hline Autores (año) & $\mathrm{N}$, diagnóstico y edad & $\begin{array}{l}\text { Instrumentos } \\
\text { de evaluación }\end{array}$ & Intervención & Diseño & Resultados \\
\hline $\begin{array}{l}\text { Zylowska et al. } \\
(2008)\end{array}$ & $\begin{array}{l}\mathrm{N}=32 \text { ( } 24 \text { adultos con una media } \\
\text { de edad de } 48.5 \text { y } 8 \\
\text { adolescentes con una media de } \\
\text { edad de } 15.6 \text { ) } \\
\text { Todos con diagnóstico de } \\
\text { TDAH, no grupo de control. }\end{array}$ & $\begin{array}{l}\text { BAI } \\
\text { BDI } \\
\text { CDI } \\
\text { RMCAS } \\
\text { ANT }\end{array}$ & $\begin{array}{l}\text { Adaptación de Mindulness } \\
\text { de Zylowska (MAPs). } \\
\text { Programa de } 8 \text { semanas de } \\
\text { intervención. }\end{array}$ & $\begin{array}{l}\text { Aplicación el programa de } 8 \\
\text { semanas sin seguimiento } \\
\text { posterior. }\end{array}$ & $\begin{array}{l}\text { Se observaron mejoras pre-post en } \\
\text { atención, inhibición cognitiva, } \\
\text { ansiedad y síntomas depresivos. } \\
\text { La mayoría de los participantes } \\
\text { completaron el entrenamiento y } \\
\text { reportaron una satisfacción elevada. }\end{array}$ \\
\hline $\begin{array}{l}\text { Singh et al. } \\
(2010)\end{array}$ & $\begin{array}{l}\mathrm{N}=4 \text { ( } 2 \text { niños con TDAH de } 10 \text { y } \\
12 \text { años con medicación }+2 \\
\text { madres). } \\
\text { No grupo de control. }\end{array}$ & $\begin{array}{l}\text { SSIMC } \\
\text { SUHMC }\end{array}$ & $\begin{array}{l}\text { Programa basado en el libro } \\
\text { Fontana and Slack (1997) de } \\
12 \text { sesiones de duración. }\end{array}$ & $\begin{array}{l}\text { Sesiones separadas madres } \\
\text { e hijos. Las madres recogen } \\
\text { información en una PDA } \\
\text { diariamente sobre el } \\
\text { cumplimiento de sus } \\
\text { demandas. Línea base } 4 \\
\text { semanas antes y } \\
\text { seguimiento durante } 24 \\
\text { semanas. }\end{array}$ & $\begin{array}{l}\text { En los dos casos mejora el } \\
\text { cumplimiento de los requerimientos } \\
\text { de las madres. La interacción entre } \\
\text { hijos y madres también mejora. }\end{array}$ \\
\hline $\begin{array}{l}\text { Smalley et al. } \\
(2009)\end{array}$ & $\begin{array}{l}\mathrm{N}=105 \text { (51 con TDAH y } 54 \\
\text { grupo control) } \\
56 \text { mujeres y } 49 \text { hombres con } \\
\text { una media de edad de } 43.1 .\end{array}$ & $\begin{array}{l}\text { KIMS } \\
\text { TCl }\end{array}$ & $\begin{array}{l}\text { Entrenamiento en } \\
\text { mindfulness. }\end{array}$ & $\begin{array}{l}\text { Aplicación del entrenamiento } \\
\text { sin seguimiento posterior. }\end{array}$ & $\begin{array}{l}\text { El estudio concluye que el } \\
\text { mindfulness es una intervención } \\
\text { efectiva para personas con TDAH. }\end{array}$ \\
\hline $\begin{array}{l}\text { Van de Weijer- } \\
\text { Bergsma et al. } \\
(2012)\end{array}$ & $\begin{array}{l}\mathrm{N}=10 \text { (adolescentes con TDAH } \\
\text { entre } 11 \text { y } 15 \text { años) } \\
19 \text { padres y } 7 \text { tutores } \\
\text { No grupo control. }\end{array}$ & $\begin{array}{l}\text { BRIEF } \\
\text { CBCL } \\
\text { MAAS } \\
\text { PSI } \\
\text { YSR } \\
\text { TRF } \\
\text { SHS } \\
\text { FSS } \\
\text { ANT }\end{array}$ & $\begin{array}{l}\text { Programa MYmind basado } \\
\text { en MBTC de } 8 \text { semanas de } \\
\text { duración. }\end{array}$ & $\begin{array}{l}\text { 5adolescentes +madres } \\
\text { 4adolescentes+padres } \\
1+\text { padre+madre } \\
\text { Pre-test } 1 \text { semana antes, } \\
\text { Post-test tras las } 8 \text { semanas } \\
\text { de tratamiento. Seguimiento } \\
\text { a las } 8 \text { y } 16 \text { semanas } \\
\text { después del post-test. }\end{array}$ & $\begin{array}{l}\text { Los adolescentes y los padres } \\
\text { coinciden en una reducción en los } \\
\text { problemas de atención, en la } \\
\text { internalización y externalización de } \\
\text { problemas y mejoras en las } \\
\text { funciones ejecutivas. No coinciden } \\
\text { en estas mejoras madres y tutores. }\end{array}$ \\
\hline $\begin{array}{l}\text { Van der Oord et } \\
\text { al. (2012) }\end{array}$ & $\begin{array}{l}\mathrm{N}=22 \text { (16 niños y } 6 \text { niñas con } \\
\text { TDAH entre } 8 \text { y } 12 \text { años y sus } \\
\text { padres) } \\
\text { Grupo de control } \\
\mathrm{N}=11 \text { niños+padres }\end{array}$ & $\begin{array}{l}\text { DBDRS } \\
\text { PSI } \\
\text { PS } \\
\text { MAAS } \\
\text { ARS }\end{array}$ & $\begin{array}{l}\text { Programa MYmind basado } \\
\text { en MBTC/MBSR de } 8 \\
\text { semanas de duración. }\end{array}$ & $\begin{array}{l}\text { Pretest } 1 \text { semana antes, } \\
\text { postest al terminar la } \\
\text { intervención, seguimiento a } \\
\text { las } 8 \text { semanas de finalizar el } \\
\text { tratamiento. } \\
\text { Cuestionario profesores. }\end{array}$ & $\begin{array}{l}\text { Tanto los padres como los niños } \\
\text { refieren reducción en la } \\
\text { sintomatología TDAH. Reducción } \\
\text { del estrés parental. Los datos de los } \\
\text { profesores no son significativos } \\
\text { respecto a la reducción de síntomas } \\
\text { TDAH. }\end{array}$ \\
\hline
\end{tabular}




\begin{tabular}{|c|c|c|c|c|c|}
\hline $\begin{array}{l}\text { Carboni et al. } \\
(2013)\end{array}$ & $\begin{array}{l}\mathrm{N}=4 \text { niños con diagnóstico de } \\
\text { TDAH con } 8 \text { años de edad. } \\
\text { No grupo control. }\end{array}$ & $\begin{array}{l}\text { BASC-2-PRS } \\
\text { BASC-2-TRS } \\
\text { BOSS }\end{array}$ & $\begin{array}{l}\text { Programa modificado MSBR } \\
\text { para niños + ejercicios } \\
\text { Goleman. } \\
\text { De } 10 \text { a } 17 \text { sesiones. }\end{array}$ & $\begin{array}{l}\text { Intervención individualizada } \\
\text { a cada uno de los } 4 \text { niños, } \\
\text { sesiones de } 30 \text { a } 45 \text { minutos } \\
2 \text { veces por semana fuera de } \\
\text { clase, observación durante } \\
\text { la ejecución de tareas en } \\
\text { clase. Niños con medicación. }\end{array}$ & $\begin{array}{l}\text { Se reducen los síntomas de } \\
\text { hiperactividad y mejora la ejecución } \\
\text { de tareas en clase. }\end{array}$ \\
\hline $\begin{array}{l}\text { Haydicky et al. } \\
(2015)\end{array}$ & $\begin{array}{l}\mathrm{N}=18 \text { adolescentes con } \\
\text { diagnóstico de TDAH entre } 13 \text { y } \\
18 \text { años de edad y } 17 \text { padres. } \\
\text { No grupo de control. }\end{array}$ & $\begin{array}{l}\text { AAQ } \\
\text { Conners-3 } \\
\text { FAD } \\
\text { IC } \\
\text { IM-P RCADS } \\
\text { SIPA ADHD } \\
\text { WASI }\end{array}$ & $\begin{array}{l}\text { Program MYmind basado en } \\
\text { MBTC de } 8 \text { semanas de } \\
\text { duración. }\end{array}$ & $\begin{array}{l}\text { Línea Base (4 semanas } \\
\left.\text { antes) Pretest ( } 1^{\text {a }} \text { sesión }\right) \\
\text { Postest ( } 8^{\mathrm{a}} \text { sesión) } \\
\text { Seguimiento ( } 6 \text { semanas } \\
\text { después de la última sesión) } \\
\text { Entrenamiento paralelo a los } \\
\text { padres. No aleatorio. }\end{array}$ & $\begin{array}{l}\text { En adolescentes se reducen los } \\
\text { problemas de atención y mejora la } \\
\text { conducta entre iguales y el deterioro } \\
\text { funcional. Los padres manifiestan } \\
\text { una reducción de su nivel de estrés } \\
\text { y mayor control parental. Los } \\
\text { resultados se mantienen a las } 6 \\
\text { semanas del post-tratamiento. }\end{array}$ \\
\hline $\begin{array}{l}\text { Meppelink et al. } \\
(2016)\end{array}$ & $\begin{array}{l}\mathrm{N}=120 \text { familias de niños con } \\
\text { TDAH entre } 9 \text { y } 18 \text { años. } \\
\text { No grupo de control. }\end{array}$ & $\begin{array}{l}\text { DBDRS } \\
\text { CBCL } \\
\text { TRF } \\
\text { YSR } \\
\text { TOF } \\
\text { TEA-Ch }\end{array}$ & $\begin{array}{l}\text { Protocolo Mymind de } 8 \\
\text { semanas de duración. }\end{array}$ & $\begin{array}{l}\text { Pretest-Postest y } \\
\text { seguimiento ( } 4-10 \text { meses). } \\
\text { Estudio aleatorio ( } \mathrm{N}=60 \\
\text { grupo mindfulness, }(\mathrm{N}=60 \\
\text { medicación) Intervención } \\
\text { paralela a padres e hijos. }\end{array}$ & $\begin{array}{l}\text { La intervención mindfulness puede } \\
\text { ser una alternativa a la medicación. } \\
\text { El coste, sin embargo, no es menor } \\
\text { al menos durante las } 4 \text { semanas de } \\
\text { tratamiento. }\end{array}$ \\
\hline $\begin{array}{l}\text { Mitchell et al. } \\
(2013)\end{array}$ & $\begin{array}{l}\mathrm{N}=18 \text { adultos entre } 18 \text { y } 50 \\
\text { años. } \\
\text { Grupo control } \\
\mathrm{N}=9\end{array}$ & $\begin{array}{l}\text { CAARS } \\
\text { CAADID } \\
\text { DEFS } \\
\text { BRIEF } \\
\text { DERS } \\
\text { DTS }\end{array}$ & $\begin{array}{l}\text { Versión modificada de } \\
\text { MBCT, MAPs de } 8 \text { semanas } \\
\text { de duración }\end{array}$ & $\begin{array}{l}\text { Asignación aleatoria } \\
\text { Grupo tratamiento } \mathrm{N}=11 \text { ( } 6 \\
\text { mujeres, } 5 \text { hombres) } \\
\text { Grupo control } \mathrm{N}=9 \text { ( } 5 \\
\text { mujeres, } 4 \text { hombres) } \\
\text { Línea base, } 2 \text { semanas } \\
\text { pretratamiento, seguimiento } \\
2 \text { semanas y post- } \\
\text { tratamiento. }\end{array}$ & $\begin{array}{l}\text { Mejora en los problemas de } \\
\text { atención, hiperactividad, regulación } \\
\text { emocional y funciones ejecutivas. }\end{array}$ \\
\hline $\begin{array}{l}\text { Schoenberg et } \\
\text { al. }(2014)\end{array}$ & $\begin{array}{l}\text { Grupo TDAH } \\
N=50 \text { adultos entre } 18 \text { y } 65 \text { años } \\
\text { Grupo control } \\
\mathrm{N}=24\end{array}$ & $\begin{array}{l}\text { CAARS } \\
\text { KIMS } \\
\text { ERP análisis }\end{array}$ & $\begin{array}{l}\text { Intervención MTBC de } 12 \\
\text { semanas de duración. }\end{array}$ & $\begin{array}{l}\text { Estudio aleatorio } \\
\text { Medida ERP en tareas NO- } \\
\text { GO, errores de } \\
\text { procesamiento. }\end{array}$ & $\begin{array}{l}\text { Encontraron un incremento en la } \\
\text { amplitud de las respuestas a tareas } \\
\text { NO-GO-P3 y de los potenciales } \\
\text { evocados tras el tratamiento MTBC, } \\
\text { que se correlaciona positivamente } \\
\text { con mejoras en hiperactividad e } \\
\text { impulsividad, mayor control e } \\
\text { inhibición y mejoras en la atención. }\end{array}$ \\
\hline
\end{tabular}




\begin{tabular}{|c|c|c|c|c|c|}
\hline $\begin{array}{l}\text { Bueno et al. } \\
\text { (2015) }\end{array}$ & $\begin{array}{l}\mathrm{N}=68 \text { (hombres) } \\
43 \text { TDAH y } 25 \text { sanos } \\
\text { Grupo control } \\
\mathrm{N}=20 \text { (10 meditación, } 10 \text { no } \\
\text { intervención) } \\
\text { Entre } 18 \text { y } 45 \text { años }\end{array}$ & $\begin{array}{l}\text { BDI } \\
\text { STAI-T } \\
\text { ASRS } \\
\text { PANAS-X } \\
\text { AAQoL } \\
\text { ANT } \\
\text { CPT II }\end{array}$ & $\begin{array}{l}\text { Programa MAP Zylowska de } \\
8 \text { semanas de duración. }\end{array}$ & $\begin{array}{l}\mathrm{N}=48 \text { ( } 34 \text { con medicación, } \\
24 \text { se asignan al grupo } \\
\text { meditación, } 24 \text { no } \\
\text { intervención). }\end{array}$ & $\begin{array}{l}\text { La intervención muestra mejoras en } \\
\text { parámetros afectivos, en la calidad } \\
\text { de vida, funciones ejecutivas y de } \\
\text { atención, tanto en adultos con } \\
\text { TDAH como en los grupos control. }\end{array}$ \\
\hline $\begin{array}{l}\text { Janssen et al. } \\
(2015)\end{array}$ & $\begin{array}{l}\text { Grupo TDAH } N=120 \\
\text { Grupo control } N=60 \\
+18 \text { años }\end{array}$ & $\begin{array}{l}\text { CARRS- } \\
\text { INV:SV } \\
\text { CARRS-S:SV } \\
\text { BRIEF-A } \\
\text { FFMQ-SF } \\
\text { SCS-SF } \\
\text { MHC-SF } \\
\text { OQ } \\
\text { QALYS }\end{array}$ & $\begin{array}{l}\text { Programa basado en MBTC } \\
\text { y MAPs de } 8 \text { semanas de } \\
\text { duración. }\end{array}$ & $\begin{array}{l}\text { Asignación aleatoria a grupo } \\
\text { tratamiento habitual } \mathrm{N}=60 \text { o } \\
\text { grupo mindfulness }+ \\
\text { tratamiento habitual } \mathrm{N}=60 \text {. } \\
\text { Evaluador ciego. Línea base } \\
\text { y postest } 3,6 \text { y } 9 \text { semanas. } \\
\text { Seguimiento a } 6-9 \text { meses. }\end{array}$ & $\begin{array}{l}\text { La intervención mejora los síntomas } \\
\text { de TDAH, las funciones ejecutivas y } \\
\text { proporciona un estado mental más } \\
\text { positivo. El coste de la intervención } \\
\text { con MBTC es menor que los } \\
\text { tratamiento habituales al ser grupal. }\end{array}$ \\
\hline Lo et al. (2016) & $\begin{array}{l}\text { Grupo TDAH N=120 niños entre } \\
5 \text { y } 7 \text { años+padres } \\
\text { Grupo control } N=60\end{array}$ & $\begin{array}{l}\text { Niños: } \\
\text { ANT } \\
\text { PSI-SF } \\
\text { HRV } \\
\text { CBCL } \\
\text { Padres: } \\
\text { ASRS } \\
\text { IM-P }\end{array}$ & $\begin{array}{l}\text { Protocolo FBMI de } 8 \\
\text { semanas de duración. }\end{array}$ & $\begin{array}{l}\text { Estudio aleatorio con grupo } \\
\text { de control, } N=60 \text { FBMI } N=60 \\
\text { no intervención. Seguimiento } \\
8 \text { y } 10 \text { semanas. }\end{array}$ & $\begin{array}{l}\text { En los niños se reducen los } \\
\text { síntomas de hiperactividad y déficit } \\
\text { de atención en comparación con el } \\
\text { grupo control. Los padres } \\
\text { manifiestan menor estrés y mayores } \\
\text { habilidades parentales. }\end{array}$ \\
\hline
\end{tabular}

Note: AAQ: Acceptance and Action Questionnaire; AAQoL: Adult ADHD Quality of Life Questionnaire; ANT: Attitudes towards Neuroleptic Treatment; ARS: ADHD Rating Scale; ASRS: Adult ADHD SelfReport Scale; BAI: Beck Anxiety Inventory; BDI: Beck Depression Inventory; BRIEF: Behavior Rating Inventory of Executive Function; BSS: Behavioral Surveillance Survey; CAARS: Conners' Adult ADHD Rating Scales; CAARS-INV: Conners' Inventory; CAARSS:SV: CAARS Screening Version; CAS: Cognitive Assessment System; CBCL: Child Behavior Checklist; CDI: Children's Depression Inventory; CPRS: Conners' Parent Rating Scales; CPT: Continuous Performance Task; DBDRS: Disruptive Behavior Disorders Rating Scale; DEFS: Deficits in Executive Functioning Scale; DERS: Difficulties in Emotion Regulation Scale; DTS: Davidson Trauma Scale; ERP: Event-Related Potential; FAD: Family Assessment Device; FFMQ: Five Facet Mindfulness Questionnaire; FFS: Flinders Fatigue Scale; GSES: General Self-Efficacy Scale;HRV Parent heart rate variability; IC: Issues Checklist; IM-P: Interpersonal Mindfulness in Parenting; KIMS: Kentucky Inventory of Mindfulness Scale; MAAS: Mindful Attention Awareness Scale; MHC-SF: The Mental Health Continuum-Short Form; OQ: Outcome Questionnaire; PANAS-X: Positive and Negative Affect Schedule; PSI: Parenting Stress Index; QALY: Quality-Adjusted Life-Year; RCADS: Revised Children's Anxiety and Depression Scale; RCMAS: Revised Children's Manifest Anxiety Scale; SCS-SF: Self-Compassion Scale-Short Form; SHS. Student Health Services; SIPA: Stress index for Parents of Adolescents; STAl-T: State-Trait Anxiety Inventory; TCl: Temperament and Character Inventory; TiC-P: Trimbos/iMTA questionnaire for costs associated with psychiatric illness; TRF: Teacher's Report Form; WRI: Wender-Reimherr Interview; YSR: Youth Self Repor 


\section{Discusión}

El propósito de esta revisión es examinar las evidencias sobre la eficacia de intervenciones basadas en mindfulness como medio para mejorar la sintomatología del TDAH. Diversos trabajos corroboran que el entrenamiento en mindfulness nos ayuda a centrarnos en el momento presente y, por tanto, contribuye a desarrollar nuestro control de la atención (Baer, 2003), mejora la capacidad de atención sostenida y disminuye las distracciones (Bishop et al., 2004).

En los últimos años, algunas investigaciones han demostrado la influencia de los efectos del mindfulness en muestras no clínicas sobre las funciones ejecutivas, la atención y el autocontrol (Diamond y Lee, 2011; Flook et al., 2010).

Nuestro estudio se ha centrado concretamente en aquellos estudios realizados con muestras clínicas diagnosticadas de TDAH. Aunque debido a las características intrínsecas del trastorno podría pensarse que las personas con TDAH tendrían dificultades para seguir un programa de mindfulness, Smalley et al. (2009) demuestran que es una intervención factible para estas personas.

Como se puede comprobar en los resultados, los estudios seleccionados encuentran mejoras en diferentes aspectos de la sintomatología del TDAH, como son las dificultades de atención, la hiperactividad e impulsividad, el control emocional e incluso una reducción ante una posible ansiedad y/o depresión asociada.

La intervención con los padres de niños con TDAH es también muy importante, puesto que estos suelen experimentar mayores niveles de estrés que los padres de niños sin ningún tipo de diagnóstico. Generalmente, elevados niveles de estrés se traducen en mayor conflictividad en casa, ya que los padres suelen reaccionar en exceso ante las conductas disruptivas de los niños. Esto implica que, en algunos casos, los padres con menores diagnosticados de TDAH presenten una menor predisposición a proporcionarles apoyo. Las interacciones entre padres e hijos suelen ser más complicadas y, como consecuencia, las familias y los niños presentan una peor salud mental.

Los beneficios de incluir a las familias en la intervención son varios: por un lado, favorece la práctica diaria y el seguimiento de las intervenciones por parte de los menores en casa al conocer los propios padres el protocolo; por otro lado, los padres les proporcionan un modelo a seguir a los hijos, además de que incluir a los padres en la intervención favorece la adherencia al tratamiento. Este es un dato importante, ya que se ha comprobado que un $50 \%$ de los niños y jóvenes que acuden a terapia abandonan el tratamiento antes de finalizarlo (Nock y Ferriter, 2005).

En aquellos casos en los que los padres acuden a sesiones de mindfulness, se ha comprobado que estos manifiestan menor estrés y mayores habilidades parentales (Lo et al., 2016). En algún estudio, como en el de Weijer-Bergsma et al. (2012), se encontraron diferencias entre la percepción de mejoras entre madres y padres, siendo más significativas entre estos últimos. Esto podría deberse a que los padres suelen tener una menor presencia en casa, por lo que están menos en contacto con los niños que las madres. Este hecho puede distorsionar su percepción respecto a las mejoras obtenidas.

El nivel de cumplimiento de las intervenciones con las familias en los estudios presentados es especialmente elevado, aunque en algunos casos este seguimiento ha 
sido propiciado, en el caso de los adolescentes, con algún tipo de recompensa (Van de Weijer-Bergsma et al., 2012; Van der Oord et al., 2012).

Respecto a los beneficios del mindufulness en niños, solamente se ha seleccionado un estudio en el que la intervención se realiza exclusivamente con niños diagnosticados de TDAH (Carboni et al., 2013). En este estudio no se encuentran mejoras en atención pero sí en las tareas escolares después de la intervención en mindfulness. Hay que tener en cuenta que la muestra de este estudio es muy pequeña ya que solo se realizó con 4 menores. No obstante, es un estudio muy interesante que introduce un nuevo factor, ya que incluye instrumentos de medida objetivos y subjetivos con observaciones directas en la propia clase. Es importante continuar realizando estudios exclusivamente con menores, ya que no siempre es posible realizar una intervención paralela con los familiares.

En algunos estudios se ha hipotetizado la posibilidad de que el tratamiento basado en midfulness sea una alternativa a la medicación para aquellas personas que o bien sean reacias a tomarla, o bien no obtengan resultados positivos con la medicación o su administración les cause efectos secundarios. En el estudio Meppelink et al. (2016) se demuestra que, aunque el coste económico no sea menor, la intervención con mindfulness puede ser una alternativa o un complemento a la medicación. No obstante, a pesar de ser un estudio aleatorizado, este presenta ciertas limitaciones, ya que los participantes demostraban una fuerte preferencia hacia un tratamiento $u$ otro, lo que puede haber influido en los resultados.

Otro estudio que ha tenido en cuenta la medicación es el de Janssen et al. (2015), en el que se realiza una comparativa entre el grupo asignado a la intervención mindulness y otro grupo con medicación y/o con otro tratamiento habitual como psicoeducación. Este estudio es uno de los primeros que, además de incluir grupos aleatorizados, se trata de un experimento doble ciego, que incluye períodos de seguimiento de hasta 6 meses y se realiza en tres centros de salud diferentes especializados en tratamiento para personas con TDAH. Los resultados avalan los beneficios del mindfulness para reducir los síntomas, mejorar las funciones ejecutivas, promover un estado mental positivo y de autocompasión, además de presentar la ventaja respecto al coste de que es una intervención susceptible de realizarse en grupo.

A pesar de los efectos positivos obtenidos, la metodología de los estudios incluidos en la presente revisión es limitada por diferentes motivos: 1) ausencia de grupos de control en 7 de los 13 estudios ( Van der Oord et al., 2012, Weijer-Bergsma, et al., 2012, Zylowska et al., 2008, Carboni et al., 2013, Van de Weijer-Bergsma et al., 2012, Singh et al., 2010, Haydicky et al., 2015); 2) falta de período de seguimiento o período breve de seguimiento (Zylowska et al., 2008, Mitchell et al., 2013); 3) muestras demasiado pequeñas, de menos de 50 sujetos $\mathrm{y}$, por tanto, no representativas (Van der Oord et al., 2012, Weijer-Bergsma, et al., 2012, Zylowska et al., 2008, Carboni et al., 2013, Singh et al., 2010, Mitchell et al., 2013, Haydicky et al., 2015); 4) escasez de estudios aleatorizados (Van der Oord et al., 2012, Van de Weijer-Bergsma et al., 2012, Zylowska et al., 2008, Smalley et al., 2009, Haydicky et al., 2015, Carboni et al., 2013, Singh et al., 2010); 5) no ser conscientes del objeto de la investigación (Janssen et al., 2015, Lo et al., 2016).

La variabilidad en la duración de las intervenciones también dificulta la rigurosidad a la hora de realizar una comparativa experimental. Es importante destacar que en ninguno de los 13 estudios se han referido efectos adversos tras la intervención. Únicamente existen algunos casos de abandono por diferentes motivos. Además la adherencia al tratamiento es muy alta y en los seguimientos a largo plazo se ha detectado que los participantes, una vez finalizada la intervención, continúan practicando mindfulness. 
Este dato favorece la elección de este tipo de terapias frente a la medicación, que puede producir efectos secundarios negativos.

Futuras investigaciones deberían incluir muestras aleatorizadas, grupos de control, compasión entre diferentes modalidades de tratamiento, intervenciones con niños, adultos y tratamientos paralelos con las familias, intervenciones más amplias, períodos de seguimiento más largos, así como investigaciones con neuroimagen para identificar las zonas neurológicas activadas, pudiendo, de esta manera, demostrar los beneficios clínicos y los mecanismos de intervención de mindfulness respecto al TDAH.

Esta revisión incluye relevantes estudios sobre los efectos de las intervenciones basadas en mindfulness como tratamiento para personas con TDAH. En la mayoría de ellos encontramos efectos positivos, ya que la intervención logra reducir la sintomatología, mejora el comportamiento en niños, reduce el estrés y mejora las habilidades parentales. Las intervenciones, además, muestran beneficios secundarios como un estado mental más positivo y una mayor autocompasión. A través de estos estudios parece demostrarse el alto potencial del mindfulness como terapia principal o como complemento a otras terapias. No obstante, se requieren más estudios para replicar y expandir estos resultados.

Referencias. Los artículos incluidos en la revisión están marcados con un asterisco $\left(^{*}\right)$ :

American Psychiatric Association. (2000). Diagnostic criteria from DSM-IV. Washington, DC: Author.

American Psychiatric Association. (2013). Diagnostic and statistical manual of mental disorders (5th ed.) Washington, DC: Author.

Baer, R. A. (2003). Mindfulness training as a clinical intervention: A conceptual and empirical review. Clinical Psychology: Science and Practice, $10,125-143$.

Biederman, J., Wilens, T., Mick, E., Faraone, S. V., Weber, W., Curtis, S., ... y Soriano, J. (1997). Is ADHD a risk factor for psychoactive substance use disorders? Findings from a four-year prospective follow-up study. Journal of the American Academy of Child \& Adolescent Psychiatry, 36, 21-29.

Bishop, S. R., Lau, M., Shapiro, S., Carlson, L., Anderson, N. D., Carmody, J., ... y Devins, G. (2004). Mindfulness: A proposed operational definition. Clinical Psychology: Science and Practice, 11(3), 230-241.

Bögels, S., Hoogstad, B., van Dun, L., de Schutter, S., y Restifo, K. (2008). Mindfulness training for adolescents with externalizing disorders and their parents. Behavioural and Cognitive Psychotherapy, 36(2), 193-209.

*Bueno, V. F., Kozasa, E. H., da Silva, M. A., Alves, T. M., Louzã, M. R., y Pompéia, S. (2015). Mindfulness meditation improves mood, quality of life, and attention in adults with attention deficit hyperactivity disorder. BioMed Research International. doi: 10.1155/2015/962857

*Carboni, J. A., Roach, A. T., y Fredrick, L. D. (2013). Impact of mindfulness training on the behavior of elementary students with attentiondeficit/hyperactive disorder. Research in Human Development, 10(3), 234-251.

Daley, D., y Birchwood, J. (2010). ADHD and academic performance: why does ADHD impact on academic performance and what can be done to support ADHD children in the classroom? Child: Care, Health and Development, 36, 455-464.

Diamond, A., y Lee, K. (2011). Interventions shown to aid executive function development in children 4 to 12 years old. Science, 333(6045), 959964.

Faraone, S. V. (2003). Understanding the effect size of ADHD medications: Implications for clinical care. Medscape Psychiatry and Mental Health, $8(2)$.

Flook, L., Smalley, S. L., Kitil, M. J., Galla, B. M., Kaiser-Greenland, S., Locke, J., ... y Kasari, C. (2010). Effects of mindful awareness practices on executive functions in elementary school children. Journal of Applied School Psychology, 26(1), 70-95.

Greenfield, E., y Fischel, J. E. (2005). The impact of preschool inattention, hyperactivity, and impulsivity on social and academic development: a review. Journal of Child Psychology and Psychiatry, 46(7), 755-773.

Greenhill, L. L., Halperin, J. M., y Abikoff, H. (1999). Stimulant medications. Journal of the American Academy of Child and Adolescent Psychiatry, $38,503-512$. 
*Haydicky, J., Shecter, C., Wiener, J., y Ducharme, J. M. (2015). Evaluation of MBCT for adolescents with ADHD and their parents: Impact on individual and family functioning. Journal of Child and Family Studies, 24(1), 76-94.

Hayes, S. C., Luoma, J. B., Bond, F. W., Masuda, A., y Lillis, J. (2006). Acceptance and commitment therapy: Model, processes and outcomes. Behaviour Research Therapy, 44(1), 1-25.

Hesslinger, B., Van Elst, L. T., Nyberg, E., Dykierek, P., Richter, H., Berner, M., y Ebert, D. (2002). Psychotherapy of attention deficit hyperactivity disorder in adults: A pilot study using a structured skills training program. European Archives of Psychiatry and Clinical Neuroscience, 252(4), 177-184.

*Janssen, L., Kan, C. C., Carpentier, P. J., Sizoo, B., Hepark, S., Grutters, J., ... y Speckens, A. E. (2015). Mindfulness based cognitive therapy versus treatment as usual in adults with attention deficit hyperactivity disorder (ADHD). BMC Psychiatry, 15(1), 216.

Jensen, P. S. (2001). - Introduction-: ADHD Comorbidity and Treatment Outcomes in the MTA. Journal of the American Academy of Child \& Adolescent Psychiatry, 40(2), 134-136.

Johnston, C., y Mash, E. J. (2001). Families of children with attention-deficit/hyperactivity disorder: review and recommendations for future research. Clinical Child and Family Psychology Review, 4, 183- 207.

Kabat-Zinn, J., Massion, A. O., Kristeller, J., y Peterson, L. G. (1992). Effectiveness of a meditation-based stress reduction program in the treatment of anxiety disorders. American Journal of Psychiatry, 149(7), 936-943.

Keng, S., Smoski, M. J., y Robins, C. J. (2011). Effects of mindfulness on psychological health: A review of empirical studies. Clinical Psychology Review, 31, 1041-1056.

Linehan, M. (2003). Manual de tratamiento de los trastornos de personalidad límite. Madrid: Paidós.

*Lo, H. H., Wong, S. Y., Wong, J. Y., Wong, S. W., y Yeung, J. W. (2016). The effect of a family-based mindfulness intervention on children with attention deficit and hyperactivity symptoms and their parents: design and rationale for a randomized, controlled clinical trial (Study protocol). BMC Psychiatry, 16(1), 65

*Meppelink, R., de Bruin, E. I., y Bögels, S. M. (2016). Meditation or Medication? Mindfulness training versus medication in the treatment of childhood ADHD: a randomized controlled trial. BMC Psychiatry, 16(1), 267.

*Mitchell, J. T., Mclntyre, E. M., English, J. S., Dennis, M. F., Beckham, J. C., y Kollins, S. H. (2013). A pilot trial of mindfulness meditation training for ADHD in adulthood: impact on core symptoms, executive functioning, and emotion dysregulation. Journal of Attention Disorders. doi: 1087054713513328

Moffitt, T., y Melchior, M. (2007). Why does the worldwide prevalence of childhood attention deficit hyperactivity disorder matter? American Journal of Psychiatry, 164(6), 856-858.

Nock, M. K., y Ferriter, C. (2005). Parent management of attendance and adherence in child and adolescent therapy: A conceptual and empirical review. Clinical Child and Family Psychology Review, 8(2), 149-166.

Ortiz-Léon, S., y Jaimes-Medrano, A. L. (2007). El trastorno por déficit de atención e hiperactividad en estudiantes universitarios. Revista de la Facultad de Medicina de la UNAM, 50(12), 125-127.

Pliszka, S. (1989). Effect of Anxiety on Cognition, Behavior, and Stimulant Response in ADHD. Journal of The American Academy of Child \& Adolescent Psychiatry, 28(6), 882-887.

Polanczyk, G., De Lima, M. S., Horta, B. L., Biederman, J., y Rohde, L. A. (2007). The worldwide prevalence of ADHD: a systematic review and metaregression analysis. American Journal of Psychiatry, 164(6), 942- 948

*Schoenberg, P. L., Hepark, S., Kan, C. C., Barendregt, H. P., Buitelaar, J. K., y Speckens, A. E. (2014). Effects of mindfulness-based cognitive therapy on neurophysiological correlates of performance monitoring in adult attention-deficit/hyperactivity disorder. Clinical Neurophysiology, 125(7), 1407-1416.

Segal, Z., Williams, J., y Teasdale, J. (2006). Terapia cognitiva de la depresión basada en la consciencia plena. Un nuevo abordaje para la prevención de las recaídas (1ª Ed.) Bilbao: Descleé de Brouwer, SA.

*Singh, N. N., Singh, A. N., Lancioni, G. E., Singh, J., Winton, A. S., W., y Adkins, A. D. (2010). Mindfulness training for parents and their children with ADHD increases the children's compliance. Journal of Child and Family Studies, 19(2), 157-166.

*Smalley, S. L., Loo, S. K., Hale, T., Shrestha, A., McGough, J., Flook, L., y Reise, S. (2009). Mindfulness and attention deficit hyperactivity disorder. Journal of Clinical Psychology, 65(10), 1087-1098. 
Teasdale, J. D., Segal, Z. V., Williams, J. M., Ridgeway, V. A., Soulsby, J. M., y Lau, M. A. (2000). Prevention of relapse/recurrence in major depression by mindfulness-based cognitive therapy. Journal of Consulting and Clinical Psychology, 68(4), 615-623.

*Van de Weijer-Bergsma, E., Formsma, A. R., de Bruin, E. I., y Bögels, S. M. (2012). The effectiveness of mindfulness training on behavioral problems and attentional functioning in adolescents with ADHD. Journal of Child and Family Studies, 21(5), 775-787.

*Van der Oord, S., Bogels, S., y Peijnenburg, D. (2012). The effectiveness of mindfulness training for children with ADHD and mindful parenting for their parents. Journal of Child and Family Studies, 21(1), 139-147.

*Zylowska, L., Ackerman, D. L., Yang, M. H., Futrell, J. L., Horton, N. I., Hale, T. S., ... y Smalley, S. L. (2008). Mindfulness meditation training in adults and adolescents with ADHD: A feasibility study. Journal of Attention Disorders, 11(6), 737-746.

Zylowska, L., Smalley, S., y Schwartz, J. (2008). Mindfulness for attention deficit hyperactivity disorder (ADHD). In F. Didonna (Ed.) Clinical handbook of mindfulness. New York: Springer. 